\title{
Neurology
}

\section{Does levodopa accelerate the pathologic process in Parkinson disease brain?}

L. Parkkinen, S.S. O'Sullivan, M. Kuoppamäki, et al. Neurology; Prepublished online September 14, 2011;

DOI 10.1212/WNL.0b013e318232ab4c

This information is current as of September 15, 2011

The online version of this article, along with updated information and services, is located on the World Wide Web at:

http://www.neurology.org/content/early/2011/09/14/WNL.0b013e318232ab4c

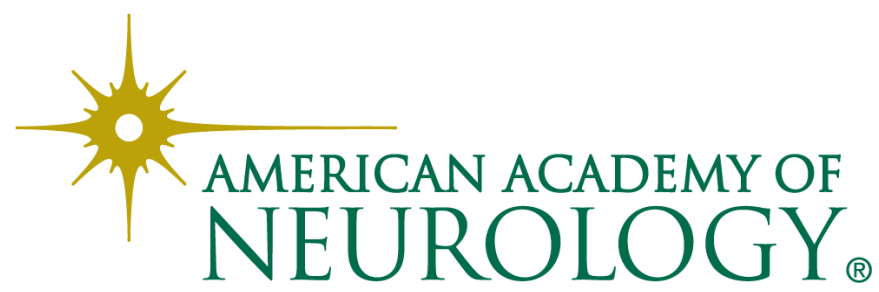




\section{Does levodopa accelerate the pathologic process in Parkinson disease brain?}

L. Parkkinen, PhD

S.S. O'Sullivan, MD, $\mathrm{PhD}$

M. Kuoppamäki, MD, $\mathrm{PhD}$

C. Collins

C. Kallis, $\mathrm{PhD}$

J.L. Holton, MD, PhD, FRCPath

D.R. Williams, MD, PhD T. Revesz, MD, FRCPath A.J. Lees, MD, FRCP

Address correspondence and reprint requests to Professor Andrew J. Lees, Reta Lila Weston Institute for Neurological Studies, UCL Institute of Neurology, 1 Wakefield Street, London WC1N 1PJ, UK

andrew.lees@ucl.ac.uk

Editorials, pages 1414 and 1416

Supplemental data at www.neurology.org

\section{ABSTRACT}

Background: Several in vitro studies have suggested levodopa (L-dopa) to be toxic to dopaminergic neurons and that it can modulate the aggregation process of $\alpha$-synuclein. We investigated the relationship between cumulative lifetime dose of L-dopa and nigral neuronal count and Lewy body (LB) pathology in Parkinson disease (PD).

Methods: Density of pigmented neurons was measured unilaterally in a single section of substantia nigra (SN) with delineation of the dorsal and ventral tiers in 96 cases of PD with welldocumented clinical records relating to antiparkinsonian drug treatment. Cortical and nigral LB densities were determined using a morphometric approach.

Results: Mean lifetime dose of L-dopa correlated significantly ( $p<0.001$ ) with duration of PD in the entire study population ( $n=96$ ) and it was not possible to disentangle their individual effect. This was not the case in a subgroup analysis of younger onset patients with a longer duration of PD ( $n=40$ ) who showed no significant correlation between L-dopa and total SN neuronal density ( $p=0.07$ ), after adjustment for duration of illness. There was, however, a lower neuronal density in the ventral $(p=0.02)$ but not in the dorsal $(p=0.27)$ tier detected with the cumulative dose of L-dopa. We found no difference in L-dopa dose between Braak PD stages ( $p=0.58)$. Furthermore, the subgroup analysis showed no relationship of $L$-dopa dose to either cortical $(p=0.47)$ or nigral ( $p=0.48$ ) LB density.

Conclusion: Chronic use of L-dopa in PD does not enhance progression of PD pathology as far as can be determined by our observations with SN neuronal counts and LB densities. Neurology ${ }^{\circledR}$ 2011;77:1420-1426

\section{GLOSSARY}

$\mathbf{C l}=$ confidence interval; $\mathbf{D A Q}=$ dopamine-quinones; $\mathbf{E R R}=$ estimated relative rate; $\mathbf{I C C}$ = intraclass correlation coefficient; LB = Lewy body; PD = Parkinson disease; QSBB = Queen Square Brain Bank; SN = substantia nigra

Although levodopa (L-dopa) is the most effective symptomatic agent for the treatment of Parkinson disease (PD), ${ }^{1}$ the possibility that high-dose chronic L-dopa treatment may be neurotoxic to dopaminergic neurones and enhance cell death has been noted. Evidence for this is mainly derived from tissue culture systems which lack glial cells, neurotrophic factors, and antioxidants and furthermore, the concentrations of L-dopa used in most of these studies have been much higher than the peak plasma concentrations measured in patients with PD. ${ }^{2}$ In vitro studies with lower L-dopa administration into glia-conditioned media with ascorbic acid, glutathione, and neurotrophic peptides have failed to demonstrate comparable toxicity with Ldopa. ${ }^{3-6}$ In vivo animal models of PD have also failed to demonstrate L-dopa toxicity to SN and have in fact suggested a neurotrophic effect on the surviving nigral neurons with an increased density of tyrosine hydroxylase-positive fibers in the striatum. ${ }^{7,8}$ In healthy primates, chronic

\footnotetext{
From the Queen Square Brain Bank for Neurological Disorders (L.P., C.C., J.L.H., D.R.W., T.R., A.J.L.) and Reta Lila Weston Institute for Neurological Studies (S.S.O., J.L.H., A.J.L.), UCL Institute of Neurology, UK; Orion Corporation Orion Pharma (M.K.), R\&D, CNS Development, Turku; Department of Neurology (M.K.), University of Turku, Turku, Finland; Forensic Psychiatry Research Unit (C.K.), Queen Mary, University of London, UK; and Van Cleef Roet Centre for Nervous Diseases (D.R.W.), Monash University, Melbourne, Victoria, Australia. L. Parkkinen is currently affiliated with the Department of Neuropathology, University of Oxford, John Radcliffe Hospital, Oxford, UK. Study funding: This work was undertaken at UCLH/UCL, which received a proportion of funding from the Department of Health's NIHR Biomedical Research Centres funding scheme. The Queen Square Brain Bank for Neurological Disorders is supported by the Reta Lila Weston Trust, the Progressive Supranuclear Palsy (Europe) Association, the Multiple System Atrophy, and the Alzheimer's Research Trust.

Disclosure: Author disclosures are provided at the end of the article.
} 


\section{Editor's Note:}

Does levodopa accelerate the pathologic process in Parkinson disease brain?

Controversy has surrounded the use of levodopa for nearly 45 years, since its introduction for treatment of Parkinson disease (PD). Initial controversy focused on whether to use levodopa early or late in the symptomatic course. With the availability of dopamine agonists, and then MAO-B inhibitors, the debate continued, with a crescendo, regarding which agent to use first, in preference to levodopa, because of perceptions regarding dyskinesia potential and hypothetical risks for actual neuronal toxicity from levodopa.

While Rajput reported no neuronal damage (nigral or otherwise) following chronic use of levodopa in essential tremor, dopa-responsive dystonia, and a few patients with PD

(Neurology 1991;41:1422-1424, Parkinsonism Relat Disord 2001;8:95-100), the study by Parkkinen et al. reported in this issue of Neurology ${ }^{\circledR}$ squarely attempts to address this issue in a large PD pathologic series. They stated they were unable to measure any levodopa-induced damage. Despite these pathologic data, the pro and con commentaries suggest that there are contingents that will continue levodopa's controversial legacy.

We invite you to join the debate on the study and pro and con commentaries. Click on "WriteClick" on Neurology's home page (www.neurology.org) for instructions.

oral administration of high-dose L-dopa was not toxic for dopaminergic neurons. ${ }^{9}$ A large, placebo-controlled L-dopa trial in de novo PD patients was carried out to determine if $\mathrm{L}$-dopa influences the rate of progression of PD. ${ }^{10}$ The results were difficult to interpret as L-dopatreated patients did clinically better (i.e., as assessed by Unified Parkinson's Disease Rating Scale) after drug withdrawal compared to those on placebo, but neuroimaging patients with SPECT showed reduced $\beta$-CIT dopamine uptake after L-dopa treatment. Despite these findings, concerns still linger in some clinicians'

\section{Figure 1 The lifetime cumulative dose of L-dopa}

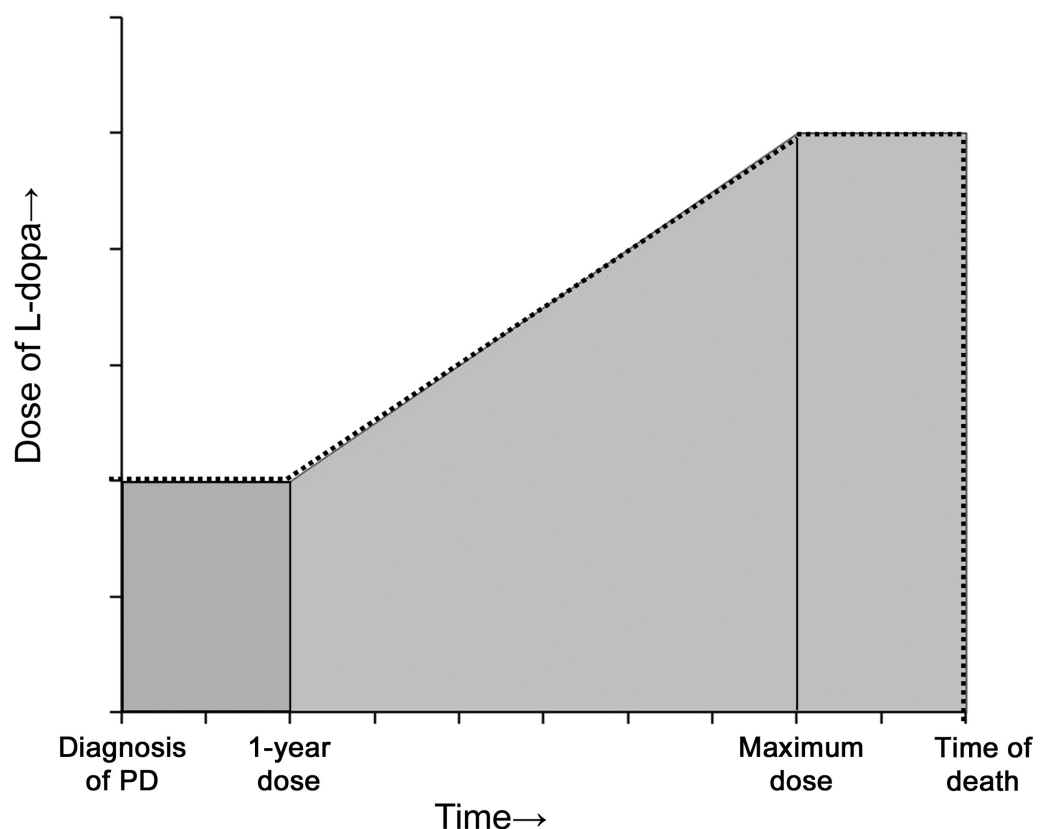

An approximation (area under the dotted line) is based on the information derived from 1-year dose, maximum dose, interval from 1-year to maximum dose, and interval from maximum dose to death. $\mathrm{PD}=$ Parkinson disease. minds about the potential neurotoxicity of the drug and as a consequence initiation of treatment may be inappropriately delayed. ${ }^{11} \mathrm{We}$ have addressed this question by examining the relationship between lifetime cumulative doses of $\mathrm{L}$-dopa and neuropathologic findings in a large well-characterized group of patients with PD.

METHODS Case selection. We identified 203 patients with an initial clinical diagnosis of parkinsonism and a pathologically proven diagnosis of $\mathrm{PD}^{12}$ from the records of donors to the Queen Square Brain Bank (QSBB) for Neurological Disorders, UCL Institute of Neurology, from the period 2000-2008. All patients had been assessed by UK hospital specialists (neurologists or geriatricians). Cases were excluded if the medical records did not contain regular and well-documented reports of the clinical symptoms and doses of pharmacologic treatments throughout the course of their illness.

Standard protocol approvals, registrations, and patient consents. All study subjects provided written informed consent to participate and London Multicenter Research Ethics Committee has approved procedures for the donation of brains to the QSBB as well as retention and access to clinical records.

Clinical assessment. The collected demographic data included gender, age at onset of PD (defined by the retrospective report of first symptoms), disease duration of PD, and age at death. Complete medication history was obtained, and an approximation of the cumulative lifetime dose of L-dopa was made based on the following equation: (daily amount of L-dopa $[\mathrm{mg}]$ at 1 year after commencement $\times 365)+1 / 2($ maximum daily amount L-dopa + daily amount L-dopa at 1 year after commencement $\times 365) \times($ interval from 1 year after commencement to reaching maximum dose in years) + (maximum daily amount L-dopa $\times 365) \times($ interval from reaching maximum dose to death in years) (figure 1). ${ }^{13}$ We also recorded the presence and duration of any dopamine agonist usage.

Since people with older disease onset with shorter survival rates may represent a more complex and aggressive phenotype with concomitant pathologies, ${ }^{14}$ we examined in further detail a more homogenous subgroup of patients with PD with "a younger onset and typical long disease duration" who have suggested to have a distribution of LB pathology which is more consistent with the Braak staging of PD. ${ }^{15}$ We included 40 patients with $\mathrm{PD}$ into this subgroup analysis with age at onset between 40 and 65 years and duration of $\mathrm{PD} \geq 15$ years, excluding 7 patients with $\mathrm{PD}$ with age at onset $<40$ years, 28 patients with age at onset $>65$ years, and 21 patients with age at onset between 40 and 65 years but duration of $\mathrm{PD}<15$ years.

Neuropathologic assessment. All brains had been fixed in $10 \%$ buffered formalin and routinely dissected according to standardized protocol used in the QSBB. ${ }^{16}$ A single, transverse $20-\mu$ m-thick section of the midbrain, taken at the level where the fascicles of the third cranial nerve emerge from the $\mathrm{SN}$, was stained with the Luxol fast blue/cresyl violet method. This is an optimal section of midbrain as it permits the evaluation of pertinent nuclear groups at that level. Single section counts of all neuromelanin-containing neurons with or without a nucleus in the SN pars compacta were obtained by using Image-Pro Plus software package (MediaCybernetics, UK). Each SN pars compacta was outlined and further divided into ventral and dorsal 
Figure 2 Transverse cut of the midbrain at the level of the third cranial nerve

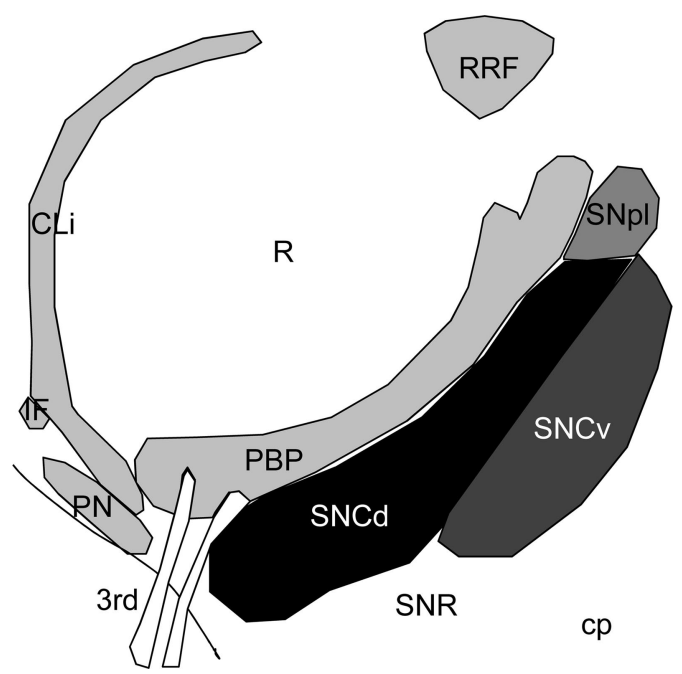

Picture shows how the 2 tiers of substantia nigra pars compacta (dorsal, SNCd and ventral, SNCv) were depicted (adapted from Halliday ${ }^{17}$ ). More ventrally positioned substantia nigra reticulata (SNR) and dorsolaterally located pars lateralis (SNpl) are also indicated. $\mathrm{CLi}=$ caudal linear nucleus; $\mathrm{cp}=$ cerebral peduncle; IF = interfascicular nucleus; $\mathrm{PBP}=$ parabrachial nucleus; $\mathrm{PN}=$ paranigral nucleus, $\mathrm{R}=$ red nucleus; $\mathrm{RRF}=$ retrorubral fields.

tiers as described in detail elsewhere (figure 2).${ }^{17}$ Pars lateralis was excluded from the analysis as in this region there is a considerable mixing of neuronal types. Software automatically divided each examined area into a number of nonoverlapping counting squares of equal size of $300 \mu \mathrm{m} \times 300 \mu \mathrm{m}$ where all pigmented neurons were counted in $\times 200$ magnification. The number of these squares was used to determine the surface area and finally the neuronal density was expressed as neurons $/ \mathrm{mm}^{2}$. The single section counting has been shown to be as reliable as the dissector method in evaluating the neuronal loss from SN.18

$\alpha S$ immunohistochemistry was carried out as described before. ${ }^{19}$ For each case, a Braak stage $(0-6)$ was assigned according to the topographic distribution of $\alpha$ S-IR inclusions. ${ }^{15}$ In addition, LBs were systematically counted by 2 investigators (L.P. and C.C.) within the total thickness of 5 cortical gray matter regions (frontal, temporal, parietal, entorhinal, and cingulate cortices) and in the $\mathrm{SN}$ and adjusted to the surface area (LB/ $\mathrm{mm}^{2}$ ) measured using Image-Pro Plus. In each case, the "total cortical LB density" was determined as the sum of counts in 5 cortical areas divided by a sum of respective surface areas. All pathologic analyses were done blinded to the clinical data.

Statistical analyses. Statistical analysis was performed using SPSS 17.0 software (SPSS Inc., Chicago, IL). Intrarater reliability for the nigral neuronal counts (25\%) and interrater reliability for all LB counts (100\%) was assessed with intraclass correlation coefficient (ICC). ICC coefficients were $>0.80$ for all the ratings performed, thus reflecting high reliability. Group comparisons were made using 2-tailed $t$ test and Kruskal-Wallis test, as appropriate. Bivariate correlations were assessed by means of Spearman coefficient. Generalized linear models were used to analyze the number of pigmented neurons and LBs. Since we were analyzing count data adjusted for area $\left(/ \mathrm{mm}^{2}\right)$ and the variances of these variables were much larger than the corresponding means (i.e., overdispersed data), we fitted the regression model based on the negative binomial distribution. The estimated relative rate (ERR) was interpreted in a similar way to the relative risk and odds ratio. Null hypothesis (i.e., cumulative L-dopa dose is not associated to nigral dopaminergic cell loss or LB formation) was rejected at the 0.05 significance level.

RESULTS Study population. Of the 203 potentially eligible cases, 107 cases were excluded due to incomplete medical records or lack of adequate brain material. The study examined 96 patients with PD who showed no significant differences in comparison to cases excluded with respect to gender (female:male ratio $26 \%: 74 \%)$, age at onset $(62.0 \pm 8.8$ years), duration of PD (14.0 \pm 5.4 years), or age at death (76.0 \pm 7.4 years). The clinical data on the entire study population $(n=96)$ and the patients of the subgroup analysis $(n=40)$ are shown in table 1 . In the entire study population, the median time interval

\section{Table 1 Relationship between the nigral neuronal density and all demographic variables ${ }^{\mathrm{a}}$}

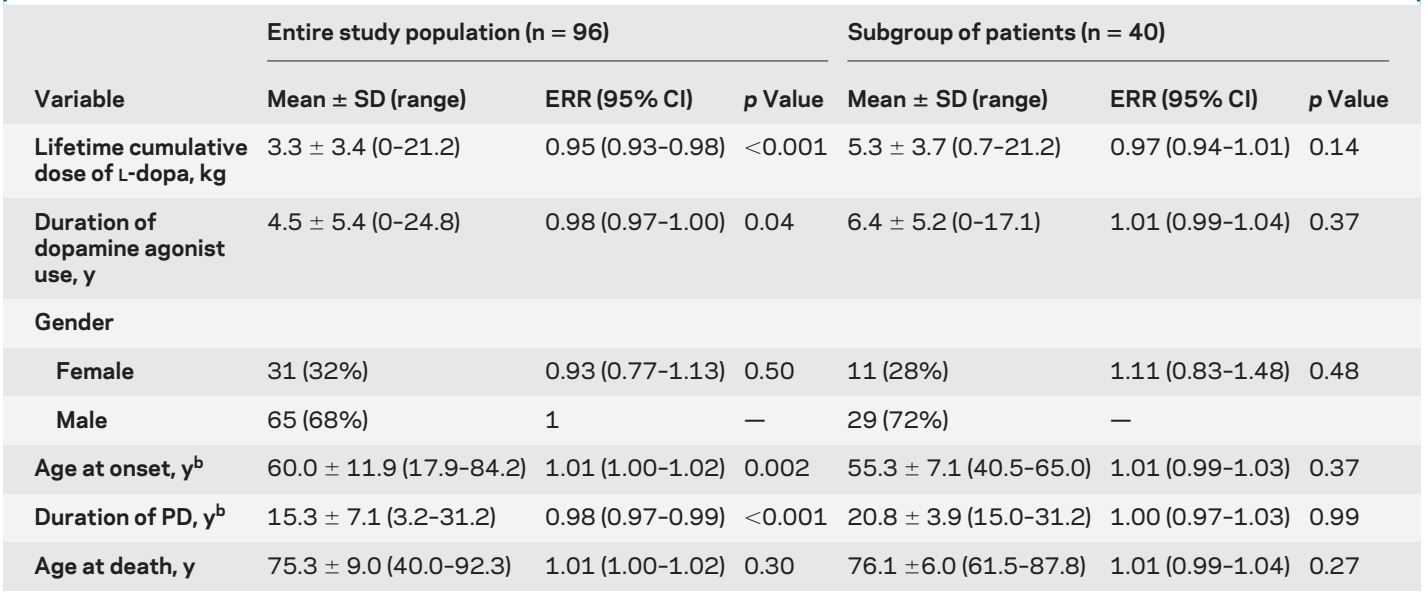

Abbreviations: $\mathrm{Cl}=$ confidence interval; $\mathrm{ERR}=$ estimated relative rate; $\mathrm{PD}=$ Parkinson disease.

a Univariate model.

b Significantly different ( $p<0.001$ ) for the cases selected to the subgroup analysis. 


\begin{tabular}{|c|c|c|c|c|c|}
\hline \multirow[t]{2}{*}{ Table 2} & \multicolumn{5}{|c|}{$\begin{array}{l}\text { Relationship between the cumulative dose of } L \text {-dopa and the nigral } \\
\text { neuronal density in the subgroup of patients }{ }^{a}\end{array}$} \\
\hline & Neuronal count $/ \mathrm{mm}^{2}$ & Mean \pm SD & Range & ERR (95\% Cl) & $p$ Value \\
\hline \multicolumn{2}{|l|}{ Total SN } & $11.4 \pm 4.5$ & $4.0-24.1$ & $0.96(0.92-1.00)$ & 0.07 \\
\hline \multicolumn{2}{|l|}{ Dorsal SN } & $13.2 \pm 4.9$ & $6.1-27.5$ & $0.98(0.94-1.02)$ & 0.27 \\
\hline \multicolumn{2}{|c|}{ Ventral SN } & $8.0 \pm 5.3$ & $0-19.1$ & $0.89(0.81-0.98)$ & 0.02 \\
\hline
\end{tabular}

Abbreviations: $\mathrm{Cl}$ = confidence interval; $\mathrm{ERR}$ = estimated relative rate; $\mathrm{SN}=$ substantia nigra.

a Multivariate model adjusted for gender, age at onset, duration of PD, and duration of dopamine agonist use.

between commencing and maximum dose of L-dopa was 6.0 years $(95 \%$ confidence interval [CI] 5.6-7.7 years) whereas the median time from reaching maximum dose to death was 4.7 years $(95 \%$ CI $4.6-6.7$ years). In the subgroup, the median interval from commencing L-dopa to maximum dose was 9.5 years (95\% CI $7.6-11.2$ years) and the median interval from maximum dose to death 7.8 years $(95 \% \mathrm{CI}$ $6.2-6.7$ years).

Relationship between the L-dopa dose and neuronal density in SN. Using univariate models (table 1), the lifetime cumulative dose of L-dopa $(p<0.001)$ and duration of PD $(p<0.001)$ were found to be negatively associated and age at onset positively associated $(p=0.002)$ with the density of pigmented neurons in the total SN. Duration of dopamine agonist use was weakly positively associated ( $p=0.04$ ), but gender and age at death were not associated with the nigral neuronal density. The results from the multivariate model showed that $\mathrm{L}$-dopa dose remained negatively associated $(p=0.03, \mathrm{ERR}=0.97,95 \%$ CI 0.94-1.00) with nigral neuronal density after adjustments for gender, age at onset, and duration of dopamine agonist use. However, as the lifetime cumulative dose of L-dopa strongly correlated with the duration of PD $(p<0.001$, Spearman rho $=0.86)$ (figure e-1 on the Neurology ${ }^{\circledR}$ Web site at www. neurology.org), their individual effects could not be separated and statistically evaluated in the entire study population. A similar strong correlation $(p<$ 0.001 , Spearman rho $=0.86$ ) between L-dopa dose and duration of $\mathrm{PD}$ was observed in 21 patients with PD with age at onset between 40 and 65 years and duration of illness $<15$ years. These patients were not included in the subgroup analysis due to their duration of illness (see Methods).

When the negative binomial modeling approach was separately applied to those patients with a more homogeneous clinical course of PD (table 1), no factors were associated with the neuronal density in SN in a univariate model. Furthermore, in this subgroup, lifetime cumulative dose of L-dopa showed only a weak correlation with duration of PD ( $p=$
0.005 , Spearman rho $=0.44$ ) (figure e-1) and therefore it could be included into our multivariate analysis. This analysis showed no significant association between the cumulative dose of L-dopa and total SN neuronal density $(p=0.07, \mathrm{ERR}=0.96,95 \%$ CI 0.92-1.00) after adjustments for the gender, age at onset, duration of $\mathrm{PD}$, and duration of dopamine agonist use (table 2). Nevertheless, when subregions of SN were analyzed separately, the neuronal density in the ventral tier $(p=0.02$, $\mathrm{ERR}=0.89,95 \%$ CI $0.81-0.98)$ but not in the dorsal $(p=0.27, \mathrm{ERR}=0.98,95 \%$ CI $0.94-$ 1.02) was significantly decreased with increasing lifetime-cumulative dose of L-dopa.

The mean SN density of pigmented neurons in the entire study population was $13.1 \pm 5.6 / \mathrm{mm}^{2}$ (range $4.0-38.2 / \mathrm{mm}^{2}$ ). Three patients were exposed to a very high cumulative dose of L-dopa $(11.4,13.0$, and $21.2 \mathrm{~kg}$ ) administered for 24, 31, and 28 years, respectively and the mean density of $\mathrm{SN}$ pigmented neurons in these patients was $10.1 \pm 3.7 / \mathrm{mm}^{2}$ (range $6.6-13.9 / \mathrm{mm}^{2}$ ). Our study population also included 2 untreated patients with PD with 4.5 and 3.2 years of $\mathrm{PD}$ duration and nigral neuronal density of 13.4 and $26.2 / \mathrm{mm}^{2}$, respectively. Due to their short survival, these 2 patients were not part of the subgroup analysis and therefore did not confound any of our results.

Relationship between the L-dopa dose and LB pathology. We found no difference in the lifetime cumulative dose of L-dopa between Braak PD stages ( $p=0.58$, Kruskal-Wallis). The mean lifetime dose of L-dopa was $3.3 \pm 2.7 \mathrm{~kg}$ in 4 patients with Braak PD stage $3,3.9 \pm 4.0 \mathrm{~kg}$ in 36 patients with stage 5 , and $3.1 \pm 2.9$ in 56 cases with stage 6 . In univariate model (table e-1), none of the examined factors were associated with the cortical LB density but the L-dopa dose $(p<0.001)$, dopamine agonist use $(p<0.001)$, and duration of PD $(p<0.001)$ were negatively and age at onset $(p=0.01)$ positively associated with the nigral LB density. However, as before, due to strong correlation between lifetime cumulative dose of L-dopa and duration of PD $(p<0.001$, Spearman rho $=0.86)$, their individual effects on nigral LB density could not be dissected in the entire sample. When the negative binomial modeling approach was separately applied to the subgroup (table 3), the univariate modeling showed a significant decrease in the LB density in the temporal cortex with increasing L-dopa dose $(p=0.03$, ERR $=0.81,95 \%$ CI $0.66-$ 0.98). This association was however not statistically significant after adjusting for gender, age at onset, duration of $\mathrm{PD}$, and duration of dopamine agonist use. The multivariate model showed that patients with PD with higher cumulative lifetime dose of 
Table 3 Relationship between the cumulative dose of L-dopa and the cortical and nigral Lewy body densities in the subgroup of patients with $P D$

\begin{tabular}{|c|c|c|c|c|}
\hline \multirow[b]{2}{*}{ Brain region $\left(\mathrm{LB} / \mathrm{mm}^{2}\right)$} & \multicolumn{2}{|l|}{ Univariate model } & \multicolumn{2}{|c|}{ Multivariate model $^{\mathrm{a}}$} \\
\hline & ERR $(95 \% \mathrm{Cl})$ & p Value & ERR (95\% CI) & p Value \\
\hline Parietal cortex & $0.66(0.49-0.88)$ & 0.05 & 0.71 (0.49-1.02) & 0.06 \\
\hline Frontal cortex & $0.86(0.69-1.07)$ & 0.17 & 0.89 (0.66-1.18) & 0.40 \\
\hline Temporal cortex & 0.81 (0.66-0.98) & 0.03 & 0.89 (0.69-1.14) & 0.35 \\
\hline Cingulate gyrus & 0.95 (0.81-1.11) & 0.51 & $0.97(0.81-1.16)$ & 0.75 \\
\hline Entorhinal cortex & $0.92(0.79-1.07)$ & 0.28 & $0.96(0.80-1.15)$ & 0.64 \\
\hline Total cortex & 0.91 (0.78-1.05) & 0.20 & $0.94(0.78-1.12)$ & 0.47 \\
\hline Substantia nigra & 0.95 (0.90-1.01) & 0.09 & 0.98 (0.91-1.04) & 0.48 \\
\hline
\end{tabular}

Abbreviations: $\mathrm{Cl}=$ confidence interval; $\mathrm{ERR}=$ estimated relative rate; $\mathrm{LB}=$ Lewy body. ${ }^{\text {a }}$ Adjusted for gender, age at onset, duration of $\mathrm{PD}$, and duration of dopamine agonist use.

L-dopa showed no difference in LB densities in any specific cortical region, the total cortical region $(p=$ $0.47)$, or SN ( $p=0.48)$. The only significant association found in the multivariate model was between LB density in the SN and duration of PD ( $p=0.04$, $\mathrm{ERR}=0.94,95 \%$ CI $0.89-1.00)$.

DISCUSSION The effects of L-dopa on the neuropathologic lesions of PD are difficult to study in a controlled setting as almost all patients now receive L-dopa treatment at some stage of their illness. The major strength of this retrospective postmortem study was the large and representative sample of patients with PD with well-documented reports of both L-dopa and dopamine agonist therapies. The potential limitation of the study was the approximation of the lifetime cumulative dose of L-dopa. L-Dopa doses are not always increased in a linear fashion and the maximum daily dose is rarely continued up until the time of death. Furthermore, reduced adherence to the prescribed regimen may occur in some patients. To try to overcome this, we used a large study population and our calculation handled patients with PD with low and high cumulative doses of L-dopa in a similar way and thus the individually calculated approximation does not create incorrect distribution (which is important for correlating L-dopa dose and neuropathologic findings).

As expected, the mean cumulative lifetime dose of L-dopa significantly correlated with the duration of $\mathrm{PD}$, and therefore, their individual effects on density of nigral neurons or LB pathology could not be evaluated for the entire study group. Nonetheless, there was no such correlation found among the patients with PD with younger age at onset and longer disease duration that have recently been described as archetypal patients with PD where the clear hierarchical pattern of LB pathology predicted by the Braak stages can be seen. ${ }^{14,15}$ In this subgroup, we found that patients with PD with higher cumulative lifetime dose of L-dopa did not show difference in the neuronal densities of total SN, after adjustment for gender, age at onset, duration of $\mathrm{PD}$, and duration of dopamine agonist therapy. We did, however, find a modest decrease in the neuronal density in the ventral but not in the dorsal tier of SN with increasing dose of L-dopa. This suggests similar regional vulnerability reported before, ${ }^{20}$ further confirming that the neuronal loss in PD is greatest and relatively selective for the ventral tier. Based on our results, L-dopa may slightly enhance this selective vulnerability, but as we found no change in the total SN number, increasing L-dopa doses may lead to less severe neuronal loss in the dorsal tier of $\mathrm{SN}$ as a compensatory effect. These results, however, should be cautiously interpreted as our observations could be entirely independent of the variables examined here. For example, increased neuromelanin content in the dorsal tier of $\mathrm{SN}$ has been suggested to function as a cytoprotective mechanism in PD. ${ }^{21}$

The mean density of SN pigmented neurons in 3 subjects who had been exposed to a very high lifetime cumulative dose of L-dopa $(>10 \mathrm{~kg})$ administered for 21 to 32 years was not significantly different from the mean $\mathrm{SN}$ density in the entire study population. No significant differences in SN density were found between these patients and 2 subjects who had never received L-dopa treatment. Although the number of these subjects is small, we believe that the overlapping $\mathrm{SN}$ neuronal densities support the main finding that cumulative lifetime L-dopa dose is not an independent risk factor for increased cell death in SN. Although the human postmortem data are limited, an autopsy study carried out a few years after L-dopa had been introduced into clinical practice found no discernible pathologic differences in general between those treated with L-dopa and those not treated. ${ }^{22}$ There is also a case report in which chronic L-dopa treatment $(2 \mathrm{~kg}$ cumulative dose over 4-year interval) did not cause nigral damage in a patient who presented features of parkinsonism..$^{23}$ Another study reported a normal SN at autopsy in one subject with essential tremor who had received $24 \mathrm{~kg}$ of L-dopa over 26 years and slight depigmentation but a normal number of nigral neurons in a further patient with dystonia who had received $3 \mathrm{~kg}$ of $\mathrm{L}$-dopa over 11 years. ${ }^{24}$

Recent in vitro evidence shows that dopaminergic neurons lacking $\alpha S$ were resistant to L-dopa-induced cell death, suggesting a complex interaction between dopamine and $\alpha S$ that eventually can lead to selective nigral degeneration. ${ }^{25} \alpha S$ has been proposed to regulate nearly every step of dopamine metabolism from biosynthesis to vesicular storage and synaptic release. ${ }^{26,27}$ Thus, a loss of functional, soluble $\alpha S$ in PD by its aggregation 
as LBs and neurites could disrupt dopamine homeostasis, ultimately leading to generation of neurotoxic dopamine-quinones (DAQ) and reactive oxygen species. Secondly, the DAQ can form covalent adducts with $\alpha$ S that stabilize toxic $\alpha$ S protofibrils by inhibiting their conversion to insoluble fibrillar aggregates. ${ }^{28-30} \mathrm{~A}$ number of studies have suggested that it is the protofibrillar form of $\alpha S$ that is responsible for the neuronal death rather than the "mature" fibrils (i.e., LBs). ${ }^{31-33}$ Furthermore, not only does dopamine inhibit $\alpha \mathrm{S}$ fibril formation but it also causes disaggregation of existing $\alpha S$ fibrils generated in vitro. ${ }^{34}$ One might expect therefore that if exogenous dopamine increased neurotoxicity by inhibiting LB formation, then patients with higher lifetime cumulative doses should show increased dopaminergic cell death in SN and overall less LB pathology. However, our results do not support such a hypothesis in regard to any difference in either total SN cell loss or extent of LB pathology. The cumulative doses of L-dopa were similar between subjects with different Braak PD stages, although as in most postmortem studies of PD, the majority of our subjects (96\%) were already in Braak PD stages 5 or $6 .{ }^{15}$ We did, however, find that LB density in the SN increased within time, suggesting that the accumulation of $\alpha S$-IR inclusions in this area is more dependent on the duration of the disease than on the cumulative dose of L-dopa. The lack of association between L-dopa dose and cortical LB density is of importance in that high cortical LB burden has been linked with cognitive impairment in PD. ${ }^{35}$

This unique clinicopathologic study examining the effect of antecedent drug exposure to the changes seen in postmortem provides further evidence that concerns over L-dopa neurotoxicity in PD patients are not warranted.

\section{AUTHOR CONTRIBUTIONS}

Dr. Lees had full access to all of the data in the study and takes responsibility for the integrity of the data and the accuracy of the data analysis. Study concept and design: Dr. Parkkinen, Dr. Kuoppamäki, Dr. Revesz, Dr. Lees. Acquisition of data: Dr. Parkkinen, Dr. O'Sullivan, C. Collins, Dr. Williams. Analysis and interpretation of data: Dr. Parkkinen, Dr. O'Sullivan, Dr. Kuoppamäki, Dr. Kallis, Dr. Holton, Dr. Revesz, Dr. Lees. Drafting of the manuscript: Dr. Parkkinen, Dr. Kuoppamäki. Critical revision of the manuscript for important intellectual content: Dr. Parkkinen, Dr. O’Sullivan, Dr. Kuoppamäki, C. Collins, Dr. Kallis, Dr. Holton, Dr. Williams, Dr. Revesz, Dr. Lees. Statistical analysis: Dr. Parkkinen, Dr. O'Sullivan, Dr. Kallis. Obtained funding: Dr. Kuoppamäki, Dr. Lees. Contribution of vital reagents/tools/patents: Dr. Holton, Dr. Revesz, Dr. Lees. Study supervision: Dr. Revesz, Dr. Lees.

\section{ACKNOWLEDGMENT}

The authors thank the patients and their families and Mikko Vahteristo for his help with the statistical review.

\section{DISCLOSURE}

Dr. Parkkinen was supported by the Orion Corporation. Dr. O'Sullivan has received funding for travel from UCB and Britannia Pharmaceuticals Ltd. Dr. Kuoppamäki has a patent pending re: Treatment for Parkinson disease and is an employee of Orion Corporation. C. Collins and Dr.
Kallis report no disclosures. Dr. Holton has received funding for travel from Merck Serono; serves on the editorial board of Neuropathology and Applied Neurobiology; and receives research support from Alzheimer's Research Trust, the Margaret Watson Memorial Trust, the Sarah Matheson Trust, Action Medical Research, Brain Net Europe, and Myositis Support Group. Dr. Williams serves on a scientific advisory board for Novartis. Dr. Revesz serves on the editorial boards for Acta Neuropathologica, Clinical Neuropathology, Neuropathology, and Applied Neurobiology and Associate Editor for the Journal of Parkinson's Disease; has received speaker honoraria from Boehringer Ingelheim; has served as a consultant for Merck Serono; and receives research support from Orion Corporation, Alzheimer's Research Trust, the European Commission, BrainNet Europe II, the Sarah Matheson Trust, the Parkinson's Disease Society, Parkinson's UK, and MRC/Wellcome Trust. Dr. Lees serves on scientific advisory boards for and has received funding for travel and speaker honoraria from Novartis, Teva Pharmaceutical Industries Ltd., MEDA Pharmaceuticals Inc., Boehringer Ingelheim, GlaxoSmithKline, Ipsen, Lundbeck Inc., Allergan, Inc., Orion Corporation, BIAL, Noscira, and Roche; serves as a consultant for Genus; and receives research support from the PSP Association and Weston Trust/The Reta Lila Howard Foundation.

Received October 27, 2010. Accepted in final form March 10, 2011.

\section{REFERENCES}

1. Cotzias GC, Van Woert MH, Schiffer LM. Aromatic amino acids and modification of parkinsonism. N Engl J Med 1967;276:374-379.

2. Olanow CW, Gauger LL, Cedarbaum JM. Temporal relationships between plasma and cerebrospinal fluid pharmacokinetics of levodopa and clinical effect in Parkinson's disease Ann Neurol 1991;29:556-559.

3. Mena MA, Pardo B, Paino CL, De Yebenes JG. Levodopa toxicity in foetal rat midbrain neurones in culture: modulation by ascorbic acid. Neuroreport 1993;4:438-440.

4. Mena MA, Casarejos MJ, Carazo A, Paíno CL, García de Yébenes J. Glia protect fetal midbrain dopamine neurons in culture from L-DOPA toxicity through multiple mechanisms. J Neural Transm 1997;104:317-328.

5. Cheng N, Maeda T, Kume T, et al. Differential neurotoxicity induced by L-DOPA and dopamine in cultured striatal neurons. Brain Res 1996;743:278-283.

6. Pardo B, Mena MA, Casarejos MJ, Paíno CL, De Yébenes JG. Toxic effects of L-DOPA on mesencephalic cell cultures: protection with antioxidants Brain Res 1995;682: 133-143.

7. Murer MG, Dziewczapolski G, Menalled LB, et al. Chronic levodopa is not toxic for remaining dopamine neurons, but instead promotes their recovery, in rats with moderate nigrostriatal lesions Ann Neurol 1998;43:561575.

8. Shin JY, Park HJ, Ahn YH, Lee PH. Neuroprotective effect of L-dopa on dopaminergic neurons is comparable to pramipexole in MPTP-treated animal model of Parkinson's disease: a direct comparison study. J Neurochem 2009;111:1042-1050.

9. Lyras L, Zeng BY, McKenzie G, Pearce RK, Halliwell B, Jenner P. Chronic high dose L-DOPA alone or in combination with the COMT inhibitor entacapone does not increase oxidative damage or impair the function of the nigro-striatal pathway in normal cynomolgus monkeys. J Neural Transm 2002;109:53-67.

10. Parkinson Study Group. Levodopa and the progression of Parkinson's disease. N Engl J Med 2004;351:2498-2508.

11. Kurlan R. "Levodopa phobia": a new iatrogenic cause of disability in Parkinson disease. Neurology 2005;64:923-924. 
12. Ince PG, Clark B, Holton JL, Revesz T, Wharton SB. Disorders of movement and system degenerations. In: Love $S$, Louis DN, Ellison DW, eds. Greenfield's Neuropathology, 8th ed. London: Edward Arnold; 2008:889-1030.

13. O'Sullivan SS, Johnson M, Williams DR, et al. The effect of drug treatment on neurogenesis in Parkinson's disease. Mov Disord 2011;26:45-50.

14. Halliday G, Hely M, Reid W, Morris J. The progression of pathology in longitudinally followed patients with Parkinson's disease. Acta Neuropathol 2008;115:409-415.

15. Braak H, Del TK, Rub U, et al. Staging of brain pathology related to sporadic Parkinson's disease. Neurobiol Aging 2003;24:197-211.

16. Trojanowski JQ, Revesz T, Neuropathology Working Group on MSA. Proposed neuropathological criteria for the post-mortem diagnosis of multiple system atrophy. Neuropathol Appl Neurobiol 2007;33:615-620.

17. Halliday G. Substantia nigra and locus coeruleus. In: Paxinos G, Mai JK, eds. The Human Nervous System, 2nd ed. London: Elsevier Academic Press; 2004:449-463.

18. Ma SY, Röyttä M, Rinne JO, Collan Y, Rinne UK. Single section and dissector counts in evaluating neuronal loss from the substantia nigra in patients with Parkinson's disease. Neuropathol Appl Neurobiol 1995;21:341-343.

19. Neumann J, Bras J, Deas E, et al. Glucocerebrosidase mutations in clinical and pathologically proven Parkinson's disease. Brain 2009;13:1783-1794.

20. Fearnley JM, Lees AJ. Ageing and Parkinson's disease: substantia nigra regional selectivity. Brain 1991;114:22832301.

21. Mori F, Nishie M, Kakita A, Yoshimoto M, Takahashi H, Wakabayashi K. Relationship among alpha-synuclein accumulation, dopamine synthesis, and neurodegeneration in Parkinson disease substantia nigra. J Neuropathol Exp Neurol 2006;65:808-815

22. Yahr MD, Wolf A, Antunes J-L, Miyoshi K, Duffy P. Autopsy findings in parkinsonism following treatment of levodopa. Neurology 1972;22:56-71.

23. Quinn N, Parkes D, Janota I, Marsden CD. Preservation of the substantia nigra and locus coeruleus in a patient receiving levodopa $(2 \mathrm{~kg})$ plus decarboxylase inhibitor over a four-year period. Mov Disord 1986;1:65-68.
24. Rajput AH, Fenton M, Birdi S, Macaulay R. Is levodopa toxic to human substantia nigra? Mov Disord 1997;12:634-638.

25. Mosharov EV, Larsen KE, Kanter E, et al. Interplay between cytosolic dopamine, calcium, and alpha-synuclein causes selective death of substantia nigra neurons. Neuron 2009;62:218-229.

26. Lotharius J, Brundin P. Pathogenesis of Parkinson's disease: dopamine, vesicles and $\alpha$-synuclein. Nat Rev Neurosci 2002;3:932-942.

27. Perez RG, Hastings TG. Could a loss of alpha-synuclein function put dopaminergic neurons at risk? J Neurochem 2004;89:1318-1324.

28. Conway KA, Rochet JC, Bieganski RM, Lansbury PT Jr. Kinetic stabilization of the alpha-synuclein protofibril by a dopamine-alpha-synuclein adduct. Science 2001;294: 1346-1349.

29. Cappai R, Leck SL, Tew DJ, et al. Dopamine promotes $\alpha \mathrm{S}$ aggregation into SDS-resistant soluble oligomers via a distinct folding pathway. FASEB J 2005;19:1377-1379.

30. Mazzulli JR, Mishizen AJ, Giasson BI, et al. Cytosolic catechols inhibit alpha-synuclein aggregation and facilitate the formation of intracellular soluble oligomeric intermediates. J Neurosci 2006;26:10068-10078.

31. Goldberg MS, Lansbury PT Jr. Is there a cause-and-effect relationship between alpha-synuclein fibrillization and Parkinson's disease? Nat Cell Biol 2000;2:E115-119.

32. Volles MJ, Lansbury PT Jr. Zeroing in on the pathogenic form of alpha-synuclein and its mechanism of neurotoxicity in Parkinson's disease. Biochemistry 2003;42:78717878.

33. Caughey B, Lansbury PT. Protofibrils, pores, fibrils and neurodegeneration: separating the responsible protein aggregates from innocent bystanders. Annu Rev Neurosci 2003;26:267-298.

34. Li J, Zhu M, Manning-Bog AB, Di Monte DA, Fink AL. Dopamine and L-dopa disaggregate amyloid fibrils: implications for Parkinson's and Alzheimer's disease. FASEB J 2004;18:962-964.

35. Hely MA, Morris JG, Reid WG, Trafficante R. Sydney Multicenter Study of Parkinson's disease: non-L-doparesponsive problems dominate at 15 years. Mov Disord 2005;20:190-199. 
Does levodopa accelerate the pathologic process in Parkinson disease brain?

L. Parkkinen, S.S. O'Sullivan, M. Kuoppamäki, et al.

Neurology; Prepublished online September 14, 2011;

DOI 10.1212/WNL.0b013e318232ab4c

This information is current as of September 15, 2011

Updated Information \& Services

Permissions \& Licensing

Reprints including high resolution figures, can be found at:

http://www.neurology.org/content/early/2011/09/14/WNL.0b01 $3 \mathrm{e} 318232 \mathrm{ab} 4 \mathrm{c}$

Information about reproducing this article in parts (figures, tables) or in its entirety can be found online at:

http://www.neurology.org/misc/about.xhtml\#permissions

Information about ordering reprints can be found online: http://www.neurology.org/misc/addir.xhtml\#reprintsus

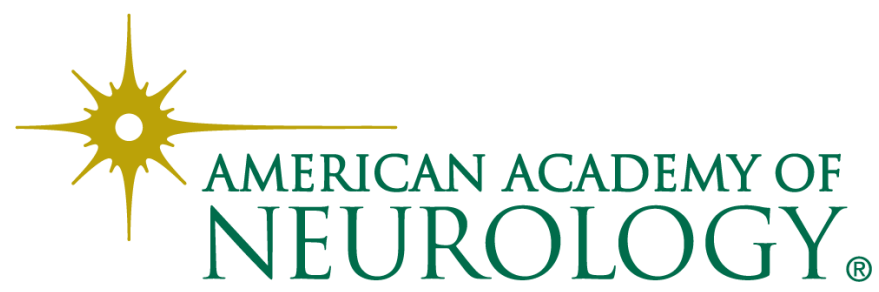

